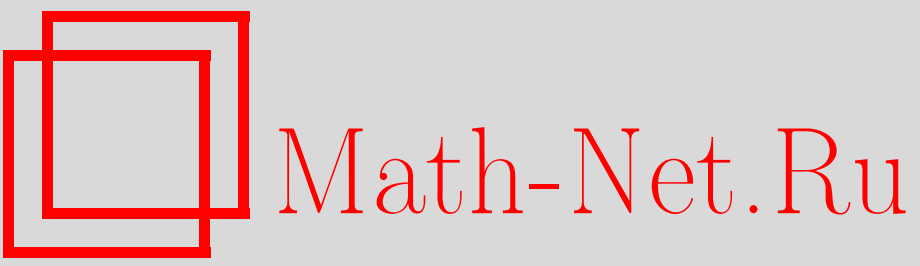

С. П. Зубова, А. Х. Мохамад, В. И. Усков, Решение полуграничной задачи для вырожденного уравнения в частных производных первого порядка, Итоги науки и техн. Сер. Соврем. мат. и ее прил. Темат. обз., 2019, том 173, 48-57

DOI: https://doi.org/10.36535/0233-6723-2019-173-48-57

Использование Общероссийского математического портала Math-Net.Ru подразумевает, что вы прочитали и согласны с пользовательским соглашением

http://www.mathnet.ru/rus/agreement

Параметры загрузки:

IP: 52.90 .164 .192

26 апреля 2023 г., 17:22:06 


\title{
РЕШЕНИЕ ПОЛУГРАНИЧНОЙ ЗАДАЧИ ДЛЯ ВЫРОЖДЕННОГО УРАВНЕНИЯ В ЧАСТНЫХ ПРОИЗВОДНЫХ ПЕРВОГО ПОРЯДКА
}

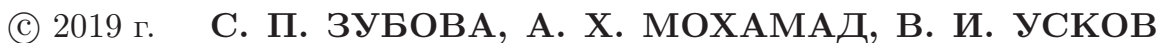

\begin{abstract}
АннотАция. Рассматривается уравнение с частными производными первого порядка в банаховом пространстве с постоянными необратимыми коэффициентами. В частном случае конечномерного пространства начально-краевая задача при необратимых матричных коэффициентах не имеет решения, поэтому в работе ставятся условия типа Шоуолтера. За счет регулярности операторного пучка уравнение расщепляется на дифференциальные уравнения в подпространствах, заданные условия приводят к начальным условиям в подпространствах. Построено решение поставленной задачи. Приведен иллюстрирующий пример.
\end{abstract}

Ключевые слова: банахово пространство, вырожденное уравнение в частных производных, 0нормальное собственное число, условия типа Шоуолтера.

\section{SOLUTION OF A SEMI-BOUNDARY-VALUE PROBLEM FOR A FIRST-ORDER DEGENERATE PARTIAL DIFFERENTIAL EQUATION}

\author{
(c) 2019 S. P. ZUBOVA, MOHAMAD ABDULFTAH HOSNI, V. I. USKOV
}

\begin{abstract}
A first-order partial differential equation with constant irreversible coefficients in a Banach space is considered. In the particular case of a finite-dimensional space, the initial-boundary-value problem with irreversible matrix coefficients has no solution; hence, we pose Showalter-type conditions. Due to the regularity of the operator pencil, the equation splits into differential equations in subspaces and given conditions lead to initial conditions in subspaces. A solution to the problem is constructed and an example is provided.
\end{abstract}

Keywords and phrases: Banach space, degenerate partial differential equation, 0-normal eigenvalue, Showalter-type conditions.

AMS Subject Classification: 35F 40

1. Введение. Рассматривается уравнение

$$
A \frac{\partial u}{\partial x}+B \frac{\partial u}{\partial y}=f(x, y),
$$

где $A: E \rightarrow E, A$-замкнутый линейный оператор, $E$-банахово пространство, $\overline{\operatorname{dom}} A=E$; число 0 для $A$ является нормальным собственным числом (см. [?]; $B \in L(E, E), B$ необратим; $f(x, y)$ - заданная непрерывная функция со значениями в $E, u=u(x, y) \in E,(x, y) \in D=$ $\left[0, x_{k}\right] \times\left[0, y_{k}\right]$. 
Под решением уравнения (1) понимается любая функция $u=u(x, y) \in C^{1}(D \rightarrow E)$, удовлетворяющая (1) при всех $(x, y) \in D$.

Впервые уравнения, неразрешенные относительно производной, изучались, по-видимому, в работе А. Пуанкаре [17]. Исследование таких уравнений, называемых дифференциально-алгебраическими, или дескрипторными, сосредоточено в нескольких математических школах: воронежская (С. Г. Крейн, А. Г. Баскаков, С. П. Зубова и др.), челябинская (Г. А. Свиридюк, В. Е. Федоров и их ученики), иркутская (Ю. Е. Бояринцев, Н. А. Сидоров, А. А. Щеглова, В. Ф. Чистяков, М. В. Фалалеев и их ученики), екатеринбургская (И. В. Мельникова и ее ученики). За рубежом активные исследования ведут A. Favini, A. Yagi, S. Campbell, P. Kunkel, V. Mehrmann, R. März, S. M. Wade, I. B. Paul и др.

Вырожденные системы уравнений в частных производных встречаются в различных приложениях: гидродинамике (уравнения Навье-Стокса; см. [13]), теплотехнике, электротехнике и т. д. Такие уравнения относят к уравнениям соболевского типа (см. [5]).

Уравнение (1), однако, нельзя назвать дифференциально-алгебраическим; далее будет показано, что уравнение (1) расщепляется на уравнения в непересекающихся подпространствах, и в каждом подпространстве уравнение дифференциальное, алгебраическая часть отсутствует. Одно из этих уравнений решается при $x=0$, другое при $y=0$; таким образом, задачу с такими условиями можно назвать и полуграничной, и полуначальной.

Заметим, конечномерный необратимый оператор $A: \mathbb{R}^{n} \rightarrow \mathbb{R}^{n}$ имеет число 0 нормальным собственным числом (см. [3]), поэтому результаты, полученные в данной работе, дополняют и уточняют соответствующие результаты работ $[1,12,14-16,18]$, полученные для случая конечномерного исходного пространства.

Свойство оператора иметь число 0 нормальным собственным числом означает, что пространство представимо в виде прямой суммы

$$
E=M \dot{+} N,
$$

где $N$ - корневое подпространство оператора $A$, а $M$ - такое инвариантное относительно $A$ подпространство, что сужение $\tilde{A}$ оператора $A$ на $M$ имеет ограниченный обратный оператор $\tilde{A}^{-1}$ (см. [4]).

Рассматривается регулярный случай, т.е. при ненулевых $\lambda$, достаточно малых по модулю $(\lambda \in \dot{U}(0))$, оператор $A-\lambda B$ обратим (пучок $A-\lambda B$ регулярный, пара $(A, B)$ регулярная) и случай одномерного ядра оператора $A$.

2. Предварительные сведения. Пусть $e_{1}, e_{2}, e_{3}, \ldots, e_{n}$-жорданова цепочка, отвечающая нулевому собственному числу оператора $A$ : $A e_{1}=0, A e_{i}=e_{i-1}, i=2,3, \ldots, n$; положим $N=\operatorname{lin}\left\{e_{1}, e_{2}, e_{3}, \ldots, e_{n}\right\}$.

В подпространстве $N$ вводится скалярное произведение $\langle\cdot, \cdot\rangle$ так, чтобы базис $e_{1}, e_{2}, e_{3}, \ldots, e_{n}$ стал ортонормированным. Проекторы на $M$ и $N$, отвечающие разложению (2), обозначаются через $Q$ и $P$ соответственно.

Приведем результаты, полученные в [6-9].

Лемма 1. Уравнение $A v=w, v, w \in E$, разрешимо относительно $v$ в том и только том случае, когда

При этом

$$
\left\langle P w, e_{n}\right\rangle=0 .
$$

əде $H=\tilde{A}^{-1} Q+S P$,

$$
v=H w+c e_{1} \quad \forall c \in \mathbb{C},
$$

$$
S=\left(\begin{array}{ccccc}
0 & 0 & \ldots & 0 & 0 \\
1 & 0 & \ldots & 0 & 0 \\
0 & 1 & \ldots & 0 & 0 \\
\vdots & \vdots & \ddots & \vdots & \vdots \\
0 & 0 & \ldots & 1 & 0
\end{array}\right)
$$

$S P: N \rightarrow N, \tilde{A}^{-1} Q: M \rightarrow M$. 
Лемма 2. Оператор $(A-\lambda B)^{-1}$ для любого $\lambda \in \dot{U}(0)$ существует тогда и только тогда, когда существует такое $q \in \mathbb{N}$, что $\left\langle P B(H B)^{q-1} e_{1}, e_{n}\right\rangle \neq 0$.

Обозначим через $p$ наименьшее из чисел $q$, для которых $\left\langle P B(H B)^{q-1} e_{1}, e_{n}\right\rangle \neq 0$.

Рассмотрим $B$-присоединенные к $e_{1}$ элементы $v_{i}$, отвечающие нулевому собственному числу:

$$
A v_{1}=0, \quad A v_{i}=B v_{i-1}, \quad i=2,3, \ldots
$$

Известно (см. [2]), что пучок $A-\lambda B$ регулярен тогда и только тогда, когда $B$-жорданова цепочка $v_{i}$ имеет конечную длину.

Лемма 3. Длина чепочки $v_{i}$ равна $p$, и в качестве $v_{i}$ можно взять элементы

$$
v_{1}=e_{1}, \quad v_{i}=(H B)^{i-1} e_{1}, \quad i=2,3, \ldots, p .
$$

Теорема 1. При $\lambda \in \dot{U}(0)$ оператор $A_{\lambda}=(A-\lambda B)^{-1}$ A имеет число 0 нормальным собственнымм числом:

$$
E=M_{1} \dot{+} N_{1},
$$

где $N_{1}$ - корневое подпространство для оператора $A_{\lambda}$, а подпространство $M_{1}$, инвариантное относительно $A_{\lambda}$, таково:

$$
M_{1}=\left\{z \in E:\left\langle P B(H B)^{i} z, e_{n}\right\rangle=0, i=1,2, \ldots, p-1 ;\left\langle P B(H B)^{p} z, e_{n}\right\rangle \neq 0\right\} .
$$

Лемма 4. Элементы подпространства $N_{1}$ являются линейными комбинациями элементов $B$-жордановой цепочки $v_{i}, i=1,2,3, \ldots, p$.

В подпространстве $N_{1}$ введем базис $\left\{v_{i}\right\}, i=1,2, \ldots, p$; в результате подпространство $N_{1}$ (как и $\left.M_{1}\right)$ не зависит от параметра $\lambda$.

Через $P_{1}$ и $Q_{1}$ обозначим проекторы на $N_{1}$ и $M_{1}$, отвечающие разложению $(7), P_{1}+Q_{1}=I$, сужение $\tilde{A}_{\lambda}$ оператора $A_{\lambda}$ на $M_{1}$ имеет ограниченный обратный оператор $\tilde{A}_{\lambda}^{-1}$.

3. Постановка задачи. Уравнение (1) расщепляется на уравнения в подпространствах $N_{1}$ и $M_{1}$, каждое из которых решается с условиями

$$
P_{1} u(x, 0)=\psi(x) \in N_{1}
$$

в подпространстве $N_{1}$ и с условиями

$$
Q_{1} u(0, y)=\varphi(y) \in M_{1} .
$$

в подпространстве $M_{1}$. Целью работы является построение решения

$$
u(x, y)=Q_{1} u(x, y)+P_{1} u(x, y)
$$

уравнения (1) с условиями типа Шоуолтера (9), (10).

4. Расщепление исходного уравнения. Представление $B=\frac{1}{\lambda}(\lambda B-A+A)$ и умножение уравнения (1) слева на $(A-\lambda B)^{-1}$ приводит к уравнению

$$
A_{\lambda} \frac{\partial u}{\partial x}+\frac{1}{\lambda}\left(A_{\lambda}-I\right) \frac{\partial u}{\partial y}=(A-\lambda B)^{-1} f(x, y) .
$$

Подставив (11) в последнее уравнение, расщепив $(A-\lambda B)^{-1} f(x, y)$ на функции в подпространствах $N_{1}$ и $M_{1}$, учитывая, что подпространства $N_{1}$ и $M_{1}$ инвариантны относительно $A_{\lambda}$ и что $N_{1} \cap M_{1}=\{0\}$, получаем уравнения

$$
A_{\lambda} \frac{\partial P_{1} u}{\partial x}+\frac{1}{\lambda}\left(A_{\lambda}-P_{1}\right) \frac{\partial P_{1} u}{\partial y}=P_{1}(A-\lambda B)^{-1} f(x, y)
$$

в подпространстве $N_{1}$ и

$$
\tilde{A}_{\lambda} \frac{\partial Q_{1} u}{\partial x}+\frac{1}{\lambda}\left(\tilde{A}_{\lambda}-Q_{1}\right) \frac{\partial Q_{1} u}{\partial y}=Q_{1}(A-\lambda B)^{-1} f(x, y)
$$

в подпространстве $M_{1}$. 
5. Решение задачи в корневом подпространстве. Для решения задачи (13), (9) элементы $P_{1} u(x, y)$ и $P_{1}(A-\lambda B)^{-1} f(x, y)$ раскладываются по базису $\left\{v_{i}\right\}, i=1,2, \ldots, p$ :

$$
\begin{aligned}
P_{1} u(x, y) & =\sum_{i=1}^{p} u_{i} v_{i}, \quad u_{i}=u_{i}(x, y), \\
P_{1}(A-\lambda B)^{-1} f(x, y) & =\sum_{i=1}^{p} F_{i} v_{i}, \quad F_{i}=F_{i}(x, y, \lambda) .
\end{aligned}
$$

Уравнение (13) принимает вид

$$
\sum_{i=1}^{p} \frac{\partial u_{i}}{\partial x} A_{\lambda} v_{i}+\frac{1}{\lambda} \sum_{i=1}^{p} \frac{\partial u_{i}}{\partial y}\left(A_{\lambda}-P_{1}\right) v_{i}=\sum_{i=1}^{p} F_{i} v_{i} .
$$

Лемма 5. Имеет место соотношение

$$
A_{\lambda} v_{i}=-\sum_{k=1}^{i-1} \frac{1}{\lambda^{i-k}} v_{k}
$$

Доказательство. Элемент $A_{\lambda} v_{i} \in N_{1}$ представим в виде

$$
A_{\lambda} v_{i}=\sum_{j=1}^{p} c_{i j} v_{j}
$$

Отсюда

$$
A v_{i}=\sum_{j=1}^{p} c_{i j}(A-\lambda B) v_{j}
$$

Элементы $A v_{j}$ заменим на элементы $B v_{j-1}, j=2,3, \ldots, p$ (равенства (5)), $A v_{1}=0$, поэтому соотношение (18) эквивалентно соотношению

$$
A v_{i}=\sum_{j=1}^{p-1}\left(c_{i j+1}-\lambda c_{i j}\right) B v_{j}-\lambda c_{i p} B v_{p}
$$

В силу леммы 1 последнее равенство корректно тогда и только тогда, когда

$$
\sum_{j=1}^{p-1}\left(c_{i j+1}-\lambda c_{i j}\right)\left\langle P B v_{j}, e_{n}\right\rangle-\lambda c_{i p}\left\langle P B v_{p}, e_{n}\right\rangle=0 .
$$

При этом

$$
v_{i}=\sum_{j=1}^{p-1}\left(c_{i j+1}-\lambda c_{i j}\right) H B v_{j}-\lambda c_{i p} H B v_{p}+c e_{1} \quad \forall c \in \mathbb{C} .
$$

В соотношении (19) $\left\langle P B v_{j}, e_{n}\right\rangle=0$ для всех $j=1,2, \ldots, p-1$ в силу (6) и определения числа $p$, поэтому $c_{i p}=0$. Теперь (20) имеет вид

$$
v_{i}=\sum_{j=1}^{p-1}\left(c_{i j+1}-\lambda c_{i j}\right) v_{j+1}+c e_{1} .
$$


Приравнивая в последнем равенстве коэффициенты при $v_{j}$ с одинаковыми нижними индексами, получаем

$$
\begin{aligned}
& \text { при } i=2 \quad c_{2 j}=0, \quad j=2,3, \ldots, p-1, \quad c_{21}=-\frac{1}{\lambda} ; \\
& \text { при } i=3 \quad c_{3 j}=0, \quad j=3,4, \ldots, p-1, \quad c_{32}=-\frac{1}{\lambda}, c_{31}=\frac{1}{\lambda} c_{32} \text {; } \\
& \text { при } i=k \quad c_{k j}=0, \quad j=k, k+1, \ldots, p-1, \quad c_{k k-1}=-\frac{1}{\lambda}, c_{k k-2}=\frac{1}{\lambda} c_{k k-1}, \ldots, c_{k 1}=\frac{1}{\lambda} c_{k 2} .
\end{aligned}
$$

Отсюда и из (17) находим

$$
A_{\lambda} v_{i}=-\frac{1}{\lambda^{i-1}} v_{1}-\frac{1}{\lambda^{i-2}} v_{2}-\cdots-\frac{1}{\lambda} v_{i-1}=-\sum_{k=1}^{i-1} \frac{1}{\lambda^{i-k}} v_{k}
$$

что и требовалось доказать.

С учетом формул (16) уравнение (15) примет вид

$$
-\sum_{i=2}^{p}\left(\frac{\partial u_{i}}{\partial x}+\frac{1}{\lambda} \frac{\partial u_{i}}{\partial y}\right) \sum_{k=1}^{i-1} \frac{1}{\lambda^{i-k}} v_{k}-\frac{1}{\lambda} \sum_{i=1}^{p} \frac{\partial u_{i}}{\partial y} v_{i}=\sum_{i=1}^{p} F_{i} v_{i} .
$$

Сравнение коэффициентов при $v_{i}$ с одинаковым нижним индексом в этом равенстве приводит к соотношениям

$$
-\frac{1}{\lambda} \frac{\partial u_{p}}{\partial y}=F_{p}, \quad-\sum_{i=1}^{j} \frac{1}{\lambda^{i}}\left(\frac{\partial u_{p-j+i}}{\partial x}+\frac{1}{\lambda} \frac{\partial u_{p-j+i}}{\partial y}\right)-\frac{1}{\lambda} \frac{\partial u_{p-j}}{\partial y}=F_{p-j}, \quad j=1,2, \ldots, p-1,
$$

откуда

$$
\frac{\partial u_{p}}{\partial y}=-\lambda F_{p}, \quad \frac{\partial u_{p-j}}{\partial y}=-\frac{\partial u_{p-j+1}}{\partial x}+F_{p-j+1}-\lambda F_{p-j}, \quad j=1,2, \ldots, p-1 .
$$

Лемма 6. Функиии $\lambda F_{p}, F_{i}-\lambda F_{i-1}, i=2,3, \ldots, p$, не зависят от $\lambda$.

Доказательство. Выражение $(A-\lambda B)^{-1} f(x, y)$, стоящее в правой части уравнения (12), представим следующим образом:

$$
(A-\lambda B)^{-1} f(x, y)=\sum_{i=1}^{p} F_{i} v_{i}+Q_{1} F, \quad Q_{1} F=Q_{1}(A-\lambda B)^{-1} f(x, y) .
$$

Отсюда

$$
f(x, y)=\sum_{i=1}^{p} F_{i}(A-\lambda B) v_{i}+(A-\lambda B) Q_{1} F .
$$

Используя определение $v_{i}$ (см. (5)), получаем:

$$
\sum_{i=1}^{p} F_{i}(A-\lambda B) v_{i}=\sum_{i=1}^{p-1}\left(F_{i+1}-\lambda F_{i}\right) B v_{i}-\lambda F_{p} B v_{p} .
$$

Следовательно, (24) переписывается в виде

$$
f(x, y)=\sum_{i=1}^{p-1}\left(F_{i+1}-\lambda F_{i}\right) B v_{i}-\lambda F_{p} B v_{p}+(A-\lambda B) Q_{1} F,
$$

откуда

$$
A Q_{1} F=f(x, y)-\sum_{i=1}^{p-1}\left(F_{i+1}-\lambda F_{i}\right) B v_{i}+\lambda F_{p} B v_{p}+\lambda B Q_{1} F
$$


Отсюда в силу леммы 1

$$
\left\langle P f, e_{n}\right\rangle=\sum_{i=1}^{p-1}\left(F_{i+1}-\lambda F_{i}\right)\left\langle P B v_{i}, e_{n}\right\rangle-\lambda F_{p}\left\langle P B v_{p}, e_{n}\right\rangle-\lambda\left\langle P B Q_{1} F, e_{n}\right\rangle,
$$

и

$$
Q_{1} F=H f(x, y)-\sum_{i=1}^{p-1}\left(F_{i+1}-\lambda F_{i}\right) H B v_{i}+\lambda F_{p} H B v_{p}+\lambda H B Q_{1} F+c e_{1} .
$$

Из последнего равенства имеем:

$$
H f(x, y)=\sum_{i=1}^{p-1}\left(F_{i+1}-\lambda F_{i}\right) H B v_{i}-\lambda F_{p} H B v_{p}+(I-\lambda H B) Q_{1} F+c e_{1} \quad \forall c \in \mathbb{C} .
$$

В правой части равенства (26) все слагаемые, кроме предпоследнего, равны нулю в силу (6)-(8) и определения числа $p$, поэтому

$$
\left\langle P f, e_{n}\right\rangle=-\lambda F_{p}\left\langle P B v_{p}, e_{n}\right\rangle .
$$

Применение к обеим частям равенства $(27)$ функционала $\left\langle P B(\cdot), e_{n}\right\rangle$ дает

$$
\left\langle P B H f, e_{n}\right\rangle=\left(F_{p}-\lambda F_{p-1}\right)\left\langle P B v_{p}, e_{n}\right\rangle-\lambda F_{p}\left\langle P B H B v_{p}, e_{n}\right\rangle .
$$

Применение к (27) функционалов $\left\langle P B(H B)^{k-1}(\cdot), e_{n}\right\rangle$ приводит к соотношениям

$$
\left\langle P(B H)^{k} f, e_{n}\right\rangle=\sum_{j=1}^{k}\left(F_{p-j+1}-\lambda F_{p-j}\right)\left\langle P B(H B)^{k-j} v_{p}, e_{n}\right\rangle-\lambda F_{p}\left\langle P B(H B)^{k} v_{p}, e_{n}\right\rangle,
$$

$k=2,3, \ldots, p-1$. Из системы $(28),(29)$ для $k=1,2, \ldots, p-1$ последовательно определяем функции $\lambda F_{p}, F_{i}-\lambda F_{i-1}, i=p-1, p-2, \ldots, 2$, не зависящие от $\lambda$, что и требовалось доказать.

Введем обозначения

$$
-\lambda F_{p}=\Phi_{p}, \quad F_{p-j+1}-\lambda F_{p-j}=\Phi_{p-j}, \quad \Phi_{p}=\Phi_{p}(x, y), \quad \Phi_{p-j}=\Phi_{p-j}(x, y),
$$

тогда уравнения (23) принимают вид

$$
\frac{\partial u_{p}}{\partial y}=\Phi_{p}, \quad \frac{\partial u_{p-j}}{\partial y}=-\frac{\partial u_{p-j+1}}{\partial x}+\Phi_{p-j}, \quad j=1,2, \ldots, p-1 .
$$

Для нахождения $P_{1} u(x, y)=\sum_{i=1}^{p} u_{i} v_{i}$ представим $\psi(x) \in N_{1}$ в соотношении (9) в виде

$$
\psi(x)=\sum_{i=1}^{p} \psi_{i}(x) v_{i}
$$

тогда

$$
u_{i}(x, 0)=\psi_{i}(x), \quad i=1,2, \ldots, p .
$$

Из первого уравнения в (30) и условия (31) с $i=p$ находим

$$
u_{p}(x, y)=\int_{0}^{y} \Phi_{p}(x, s) d s+\psi_{p}(x)
$$

из уравнения (30) с $j=1$ и условия (31) при $i=p-1$ находим

$$
u_{p-1}(x, y)=-\int_{0}^{y} \int_{0}^{\tau} \frac{\partial \Phi_{p}}{\partial x}(x, s) d s d \tau-\psi_{p}^{\prime}(x) y+\int_{0}^{y} \Phi_{p-1}(x, s) d s+\psi_{p-1}(x)
$$


при условии непрерывной дифференцируемости $\psi_{p-1}(x)$ и непрерывности второй производной по $x$ от $\Phi_{p}(x, y)$ и $\psi_{p}(x)$. Далее,

$$
u_{p-j}(x, y)=-\int_{0}^{y} \frac{\partial u_{p-j+1}(x, s)}{\partial x} d s+\int_{0}^{y} \Phi_{p-j}(x, s) d s+\psi_{p-j}(x), \quad j=1,2, \ldots, p-1 .
$$

Таким образом, последовательное решение уравнений (30) с условиями (31) приводит к следующему результату.

Лемма 7. Пусть $f(x, y)$ непрерывно дифференцируема $p+1$ раз по $x$ и $\psi(x)$ непрерывно дифферениируема $p+1$ раз. Тогда решение задачи (13), (9) существует, единственно, не зависит от $\lambda$ и определяется из соотношений (32), (33).

Замечание 1. Условия гладкости функций $f(x, y)$ и $\psi(x)$ можно ослабить за счет того, что от разных их компонент в $N_{1}$ требуется разная гладкость.

6. Решение задачи в дополнительном подпространстве. Уравнение (14) за счет обратимости оператора $\tilde{A}_{\lambda}$ в подпространстве $M_{1}$ разрешается относительно производной по $x$ :

$$
\frac{\partial Q_{1} u}{\partial x}+\frac{1}{\lambda}\left(Q_{1}-\tilde{A}_{\lambda}^{-1}\right) \frac{\partial Q_{1} u}{\partial y}=\tilde{A}_{\lambda}^{-1} Q_{1}(A-\lambda B)^{-1} f(x, y) .
$$

Правая часть этого уравнения и оператор, стоящий при производной по $y$, не зависят от $\lambda$, что и доказывается в следующих леммах.

Лемма 8. Имеет место формула

$$
\frac{1}{\lambda}\left(Q_{1}-\tilde{A}_{\lambda}^{-1}\right) Q_{1}(\cdot)=H B Q_{1}(\cdot)-\frac{\left\langle P B(H B)^{p} Q_{1}(\cdot), e_{n}\right\rangle}{\left\langle P B(H B)^{p-1} e_{1}, e_{n}\right\rangle} e_{1} .
$$

Доказательство. Введем обозначение $\frac{1}{\lambda}\left(Q_{1}-\tilde{A}_{\lambda}^{-1}\right) Q_{1} z=Q_{1} w$. Отсюда

$$
\frac{1}{\lambda}\left(\tilde{A}_{\lambda}-Q_{1}\right) Q_{1} z=\tilde{A}_{\lambda} Q_{1} w
$$

или

$$
\frac{1}{\lambda}\left((A-\lambda B)^{-1} A-I\right) Q_{1} z=(A-\lambda B)^{-1} A Q_{1} w .
$$

Умножив слева последнее равенство на $(A-\lambda B)$, получим $B\left(Q_{1} z\right)=A\left(Q_{1} w\right)$. В силу леммы 1 последнее соотношение корректно, поскольку $\left\langle P B\left(Q_{1} z\right), e_{n}\right\rangle=0$ для всех $Q_{1} z \in M_{1}$. Из леммы 1 следует также

$$
Q_{1} w=H B\left(Q_{1} z\right)+c e_{1} \quad \forall c \in \mathbb{C} .
$$

Применив к (36) функционал $\left\langle P B(H B)^{p-1}(\cdot), e_{n}\right\rangle$, получаем:

$$
0=\left\langle P B(H B)^{p} Q_{1} z, e_{n}\right\rangle+c\left\langle P B(H B)^{p-1} e_{1}, e_{n}\right\rangle .
$$

Найденное отсюда значение $c$ подставляется в (36), что и приводит к формуле (35).

Лемма 9. Функиия $\tilde{A}_{\lambda}^{-1} Q_{1}(A-\lambda B)^{-1} f(x, y)$ не зависит от $\lambda$.

Доказательство. Пусть

$$
\tilde{A}_{\lambda}^{-1} Q_{1}(A-\lambda B)^{-1} f(x, y)=g \in M_{1}
$$

тогда

$$
Q_{1}(A-\lambda B)^{-1} f(x, y)=A_{\lambda} g
$$

или

$$
(A-\lambda B) Q_{1}(A-\lambda B)^{-1} f(x, y)=A g .
$$

При доказательстве леммы 6 введено обозначение $Q_{1}(A-\lambda B)^{-1} f(x, y)=Q_{1} F$. Из (24) следует

$$
(A-\lambda B) Q_{1} F=f(x, y)-\sum_{i=1}^{p} F_{i}(A-\lambda B) v_{i},
$$


и в силу $(25)$

или

$$
(A-\lambda B) Q_{1} F=f(x, y)-\sum_{i=1}^{p-1}\left(F_{i+1}-\lambda F_{i}\right) B v_{i}-\lambda F_{p} B v_{p}
$$

$$
f(x, y)-\sum_{i=1}^{p-1}\left(F_{i+1}-\lambda F_{i}\right) B v_{i}-\lambda F_{p} B v_{p}=A g .
$$

Из леммы 6 следует, что левая часть этого равенства не зависит от $\lambda$, так что $g=g(x, y)$.

Решение задачи (34), (10) опирается на спектральные свойства линейного ограниченного оператора (см. [11]).

Пусть $\Gamma$ - замкнутый спрямляемый жорданов контур, окружающий спектр ограниченного оператора

Построим оператор

$$
y Q_{1}-(x-r) \frac{1}{\lambda}\left(Q_{1}-\tilde{A}_{\lambda}^{-1}\right), \quad 0<r<x_{k}
$$

$$
R(\mu, r) \equiv\left((y-\mu) Q_{1}-(x-r) \frac{Q_{1}-\tilde{A}_{\lambda}^{-1}}{\lambda}\right)^{-1}
$$

Решение задачи (34), (10) имеет вид

$$
Q u(x, y)=-\frac{1}{2 \pi i} \oint_{\Gamma} R(\mu, 0) \varphi(\mu) d \mu-\frac{1}{2 \pi i} \oint_{\Gamma} \int_{0}^{x} R(\mu, r) \tilde{A}_{\lambda}^{-1} Q_{1}(A-\lambda B)^{-1} f(r, \mu) d r d \mu,
$$

в чем нетрудно убедиться непосредственной подстановкой выражения (37) в (34), (10). Итак, получен следующий результат.

Теорема 2. При выполнении условий леммы 7 решение задачи (1), (9), (10) существует, единственно в корневом подпространстве и определяется формулами (32), (33) и (37).

Замечание 2. Число $p$ есть так называемый индекс (см. [15-18]) дифференциального уравнения

$$
A \frac{\partial u}{\partial x}=B u(x)+f(x)
$$

с необратимой $(n \times n)$-матрицей $A$. Иными словами, это длина $B$-жордановой цепочки присоединенных элементов для $A$, отвечающей нулевому собственному числу, или «глубина» корневого подпространства оператора $(A-\lambda B)^{-1} A, \lambda \in \dot{U}(0)$. В этом корневом подпространстве уравнение (38) алгебраическое, а в прямом дополнении - дифференциальное.

Уравнение (1), рассматриваемое в данной статье, в этом корневом подпространстве является дифференциальным с производными по $y$, а в прямом дополнении к нему - дифференциальным с производными по $x$.

7. Пример. В пространстве $\ell_{2}$ решается задача для системы

$$
\frac{\partial u_{2}}{\partial y}=0, \quad \frac{\partial u_{2}}{\partial x}+\frac{\partial u_{1}}{\partial y}+\frac{\partial u_{3}}{\partial y}=0, \quad \frac{\partial u_{3}}{\partial x}+\frac{\partial u_{4}}{\partial y}=0, \quad \ldots, \quad \frac{\partial u_{i}}{\partial x}+\frac{\partial u_{i+1}}{\partial y}=0, i=4,5, \ldots
$$

с условиями (9), (10).

Пучок $A-\lambda B$ регулярен, так как цепочка $v_{1}, v_{2}, \ldots$, для которой $A v_{1}=0, A v_{i}=B v_{i-1}$, $i=2,3, \ldots$, конечна: $v_{1}=(1,0,0, \ldots), v_{2}=(0,1,0, \ldots)$ и не существует $v_{3}$. Следовательно, $\operatorname{dim} N_{1}=2$.

Вычисления приводят к разложению $\ell_{2}=M_{1} \dot{+} N_{1}$, а именно,

$$
\left(\begin{array}{c}
u_{1} \\
u_{2} \\
u_{3} \\
u_{4} \\
\vdots
\end{array}\right)=\left(\begin{array}{c}
-u_{3} \\
0 \\
u_{3} \\
u_{4} \\
\vdots
\end{array}\right)+\left(\begin{array}{c}
u_{1}+u_{3} \\
u_{2} \\
0 \\
0 \\
\vdots
\end{array}\right)
$$


здесь первый вектор справа принадлежит $M_{1}$, второй $-N_{1}$. Условие (9) таково:

$$
u_{1}(x, 0)+u_{3}(x, 0)=\psi_{1}(x), \quad u_{2}(x, 0)=\psi_{2}(x),
$$

а условие $(10)-$

$$
u_{i}(0, y)=\varphi_{i}(y), \quad i=3,4, \ldots
$$

Система в $N_{1}$, т.е. система (23), имеет вид

$$
\frac{\partial u_{2}}{\partial y}=0, \quad \frac{\partial\left(u_{1}+u_{3}\right)}{\partial y}=-\frac{\partial u_{2}}{\partial x}
$$

откуда с учетом условий (40) определяются функции

$$
u_{2}(x, y)=u_{2}(x)=\psi_{2}(x), \quad u_{1}(x, y)+u_{3}(x, y)=\psi_{1}(x)-\psi_{2}^{\prime}(x) y .
$$

Решение задачи в $M_{1}$, построенное по формуле (37), таково:

$$
u_{i}(x, y)=-\frac{1}{2 \pi i} \sum_{j=0}^{\infty} x^{j} \oint_{\Gamma} \frac{\varphi_{i+j}(\mu)}{(y-\mu)^{j+1}} d \mu
$$

где $\Gamma$ - окружность радиуса 1 с центром в начале координат.

Если дополнительно предположить существование непрерывных производных от $\varphi_{k}(y)$ порядка $k-2, k=3,4, \ldots$, то из (43) следует

$$
u_{i}(x, y)=\sum_{j=0}^{\infty} \frac{x^{j}}{j !} \varphi_{i+j}^{(j)}(y), \quad i=3,4, \ldots
$$

Окончательно, решение задачи (39), (40), (41) имеет следующий вид:

$$
\begin{aligned}
& u_{1}(x, y)=\psi_{1}(x)-\psi_{2}^{\prime}(x)-\varphi_{3}(y)+\frac{1}{2 \pi i} \sum_{j=1}^{\infty} x^{j} \oint_{\Gamma} \frac{\varphi_{j+3}(\mu)}{(y-\mu)^{j+1}} d \mu, \\
& u_{2}(x, y)=\psi_{2}(x), \\
& u_{i}(x, y)=\varphi_{i}(y)-\frac{1}{2 \pi i} \sum_{j=1}^{\infty} x^{j} \oint_{\Gamma} \frac{\varphi_{i+j}(\mu)}{(y-\mu)^{j+1}} d \mu, \quad i=3,4, \ldots
\end{aligned}
$$

\section{СПИСОК ЛИТЕРАТУРЫ}

1. Болринцев Ю. Е. Регулярные и сингулярные системы линейных обыкновенных дифференциальных уравнений. - Новосибирск: Наука, 1980.

2. Вайнберг M. М., Треногин В. А. Теория ветвления решений нелинейных уравнений. - М.: Наука, 1969.

3. Гельфанд И. М. Лекции по линейной алгебре. - М.: МЦНМО, 1998.

4. Гохберг И. Ц., Крейн М. Г. Введение в теорию линейных несамосопряженных операторов. - М.: Наука, 1965.

5. Демиденко Г. В., Успенский С. В. Уравнения и системы, не разрешенные относительно старшей производной. - Новосибирск: Научная книга, 1998.

6. Зубова С. П. Сингулярное возмущение линейных дифференциальных уравнений, неразрешенных относительно производной/ Дисс на соиск. уч. степ. канд. физ.-мат. наук - Воронеж, 1973.

7. Зубова С. П. Свойства возмущенного фредгольмовского оператора// в кн.: Решение дифференциального уравнения с фредгольмовским оператором при производной. - Воронеж: ВГУ, 1991.

8. Зубова С. П. Решение задачи Коши для двух дифференциально-алгебраических уравнений с фредгольмовым оператором// Диффер. уравн. - 2005. - 41, № 10. - С. 1410-1412.

9. Зубова С. П. Решение однородной задачи Коши для уравнения с нетеровым оператором при производной// Докл. РАН. - 2009. - 428, № 4. - С. 444-446.

10. Зубова С. П. Чернышов К. И. О линейном дифференциальном уравнении с фредгольмовым оператором при производной// в кн.: Дифференциальные уравнения и их применение. - Вильнюс: Ин-т физ. мат. АН Лит. ССР, 1976. - 14. - С. 21-39. 
11. Крейн С. Г. Линейные дифференциальные уравнения в банаховом пространстве. - М.: Наука, 1967.

12. Нгуен Хак Диеп, Чистлков $B$. Ф. О моделировании с использованием дифференциальноалгебраических уравнений в частных производных// Вестн. ЮУрГУ. Сер. Мат. модел. програм. 2013. - 6, № 1. - С. 98-111.

13. Соболев С. Л. Об одной новой задаче математической физики// Изв. АН СССР. Сер. мат. - 1954. 18, № 1. - C. 3-50.

14. Чистлков В. Ф., Щеглова А. А. Избранные главы теории алгебро-дифференциальных систем. Новосибирск: Наука, 2003.

15. Campbell S. L. Singular Systems of Differential Equations. - London: Pitman, 1980.

16. Kunkel P., Mehrmann V. Differential-Algebraic Equations: Analysis and Numerical Solutions. - Zürich: EMA, 2006.

17. Poincaré H. Sur l'equilibre d'une masse fluide animee d'un movement de rotation// Acta Math. — 1885. - 7. - P. 259-380.

18. Wade S. M., Paul I. B. A differentiation index for partial differential algebraic equations// SIAM J. Sci. Comp. - 2000. - 21, № 6. - P. 2295-2316.

Зубова Светлана Петровна

Воронежский государственный университет

E-mail: spzubova@mail.ru

Мохамад Абдулфтах Хосни

Воронежский государственный университет

E-mail: abdulftah.hosni90@gmail.com

Усков Владимир Игоревич

Воронежский государственный лесотехнический университет им. Г.Ф. Морозова

E-mail: vum1@yandex.ru 